\title{
Research Article \\ Structural Synthesis for Prescribed Target Natural Frequencies and Mode Shapes
}

\author{
J. A. Hernandes ${ }^{1}$ and A. Suleman ${ }^{2}$ \\ ${ }^{1}$ Instituto Tecnológico de Aeronáutica, 12228-900 São José dos Campos, SP, Brazil \\ ${ }^{2}$ Department of Mechanical Engineering, University of Victoria, Victoria, BC, Canada
}

Correspondence should be addressed to J. A. Hernandes; hernandes@ita.br

Received 8 August 2013; Accepted 7 February 2014; Published 4 June 2014

Academic Editor: Miguel M. Neves

Copyright (C) 2014 J. A. Hernandes and A. Suleman. This is an open access article distributed under the Creative Commons Attribution License, which permits unrestricted use, distribution, and reproduction in any medium, provided the original work is properly cited.

\begin{abstract}
A new method for the synthesis of structures with prescribed target natural frequencies and mode shapes is presented. The introduction of a modal Rayleigh quotient approximation based on the target mode shapes is the means to propose a structural synthesis problem whose solution is free from eigenvector sensitivity analysis. The frequencies and mode shapes can be adjusted as close as possible to the desired target values, while minimizing the total mass. Several examples corroborate the efficacy of the proposed method.
\end{abstract}

\section{Introduction}

Optimization of dynamic systems is an inverse eigenvalue problem with the goal of tailoring the response of a system by estimating the needed change in the design variables to achieve a desired change in the natural frequencies of the structure. The structural synthesis with the inclusion of inequality frequency constraints has been extensively explored in the past in many different works reported in the literature [1]; however the need remains to create an effective method to include not only prescribed frequencies but also prescribed mode shapes in the optimization process. There are important instances where the adjusting of frequencies and mode shapes to given prescribed values is desirable, such as the synthesis of aircraft under aeroelastic constraints or model updating, where an analytical or numerical model is improved based on modal test data $[2,3]$.

Structural design tailoring applied to simple mass and spring systems has been extensively reported in the literature. For instance, Yee and Tsuei $[4,5]$ have proposed methods of shifting the desired eigenfrequencies using the forced response of the system and modal analysis. Mass matrix modification has been proposed to achieve desired natural frequencies [6] while the use of concentrated mass elements to shift eigenfrequencies of a rectangular plate has been proposed in [7]. Sivan and Ram [8] have used orthogonality principles to obtain modified stiffness and mass matrices. Gladwell [9] introduced an inverse approach for both the discrete and continuous structures. Mottershead [10] has investigated the concept of resonance and adding mass or springs to the dynamic system.

In other related researches, studies have been reported on the simultaneous assignment of mode frequency and shape, by developing approaches based on both modal data [11] and system physical models [12]. Examples include the assignment of natural frequencies to a multi-degree-of-freedom undamped system using an added mass connected by springs [13], the assignment of natural frequencies and antiresonances by beam modification [14], and the structural modification of a helicopter tail cone [15]. Gladwell and Movahhedy [16] obtained the set of necessary and sufficient conditions to ensure positive mass and stiffness parameters for a three-degree-of-freedom case study.

In Richiedei et al. [17], a convex optimization approach is proposed. A linear matrix difference equation is set based on satisfying the Rayleigh quotient using linearizations of the system stiffness and mass matrices in terms of the design variables. The residual of the resulting system of linear equations 
in the design variables is then used to form a convex objective function. The method can deal with general finite element models and has no need of eigenvector sensitivities but is limited in the context of structural synthesis, since it is indifferent to the structural and nonstructural mass mobilized in the solution, thus presenting a difficulty of integration with a general structural synthesis tool; besides it can only deal with linear constraints.

Although it may be immediate to write the statement of an optimization problem where the mode shapes are prescribed, the solution of such a problem may not be an easy task, since directional optimization demands eigenvector sensitivity analysis, which is expensive. Also, and perhaps mainly, numerical difficulties may arise due to complex modal structural behavior of the system, a common feature of many real life finite element discretizations.

A theoretically consistent method for the structural synthesis with prescribed target frequencies and mode shapes seems to be inexistent in literature. If such a method could avoid the use of expensive eigenvector sensitivity analysis, it might be used with great advantage. The objective of the present work is to create an effective method with strong convergence capability for the structural synthesis of general systems with prescribed target free vibration natural frequencies $\left(\bar{\omega}_{j}, j=1, \ldots, p\right)$ and mode shapes $\left(\overline{\boldsymbol{\varphi}}_{j}, j=1, \ldots, p\right)$, without the use of eigenvector sensitivities. While it is not mandatory, it is also desirable for the structure to have minimum mass.

\section{Synthesis Based on Modal Rayleigh Quotient Approximation}

The natural free vibration eigenproblem for a discrete system can be written as follows:

$$
\left(\mathbf{K}-\lambda_{j} \mathbf{M}\right) \boldsymbol{\varphi}_{j}=\mathbf{0} \quad j=1, \ldots, n,
$$

where $\lambda_{j}=\omega_{j}^{2}$ is the eigenvalue corresponding to the natural frequency $\omega_{j}$. The natural frequencies are related to corresponding mode shapes by means of the Rayleigh quotient, such that

$$
\lambda_{j}=\omega_{j}^{2}=\frac{\boldsymbol{\varphi}_{j}^{T} \mathbf{K} \boldsymbol{\varphi}_{j}}{\boldsymbol{\varphi}_{j}^{T} \mathbf{M} \boldsymbol{\varphi}_{j}} \quad j=1, \ldots, n .
$$

The Rayleigh quotient is classically used to approximate the fundamental frequency of a system and also in algorithms for solution of eigenproblems.

In this work there are $s$ prescribed pairs of target natural frequencies and mode shapes, $\left(\bar{\omega}_{j}, \overline{\boldsymbol{\varphi}}_{j}\right)$, that are to be imposed on the structure. Therefore, we seek an optimal solution $\mathbf{x}^{*}$ that will satisfy

$$
\left(\mathbf{K}^{*}-\bar{\lambda}_{j} \mathbf{M}^{*}\right) \overline{\boldsymbol{\varphi}}_{j}=\mathbf{0} \quad j=1, \ldots, s,
$$

where the eigenvalue $\bar{\lambda}_{j}=\bar{\omega}_{j}^{2}$. The target eigenvalues are related to the target mode shapes by the Rayleigh quotient:

$$
\bar{\lambda}_{j}=\frac{\overline{\boldsymbol{\varphi}}_{j}^{T} \mathbf{K}^{*} \overline{\boldsymbol{\varphi}}_{j}}{\overline{\boldsymbol{\varphi}}_{j}^{T} \mathbf{M}^{*} \overline{\boldsymbol{\varphi}}_{j}} \quad j=1, \ldots, s .
$$

The symbol ${ }^{*}$ indicates that the stiffness and mass matrices are computed at the optimal solution. It may be possible to satisfy (3) and (4) if we have the appropriate parameters or design variables to adjust in the system we are dealing with.

We define a modal Rayleigh quotient approximation (MRQA), based on the target mode shapes and the current structural stiffness and mass as follows:

$$
\mu_{j}=\frac{\overline{\boldsymbol{\varphi}}_{j}^{T} \mathbf{K} \overline{\boldsymbol{\varphi}}_{j}}{\overline{\boldsymbol{\varphi}}_{j}^{T} \mathbf{M} \overline{\boldsymbol{\varphi}}_{j}} \quad j=1, \ldots, s .
$$

The MRQA quantifies the approximation to the target mode shape $\overline{\boldsymbol{\varphi}}_{j}$ at the current design, where $\mathbf{K}$ and $\mathbf{M}$ are supposedly calculated. This quantity has in it a great deal of the dynamical structural behavior of the system and can be very helpful.

The MRQA can be used to define the following first statement of our synthesis problem, where we want to generate a structure that will have its eigenvalues and mode shapes the closest possible to the prescribed target pairs $\left(\bar{\lambda}_{j}, \overline{\boldsymbol{\varphi}}_{j}\right), j=$ $1, \ldots, s$ as follows:

$$
\begin{gathered}
\min \sum_{j=1}^{p}\left\{a_{j}\left(\mu_{j}-\bar{\lambda}_{j}\right)^{2}+b_{j}\left(\lambda_{j}-\bar{\lambda}_{j}\right)^{2}\right\} . \\
\text { s.t. } \quad \mu_{j}=\frac{\overline{\boldsymbol{\varphi}}_{j}^{T} \mathbf{K} \bar{\varphi}_{j}}{\overline{\boldsymbol{\varphi}}_{j}^{T} \mathbf{M} \overline{\boldsymbol{\varphi}}_{j}} \quad j=1, \ldots, s, \\
\left(\mathbf{K}-\lambda_{j} \mathbf{M}\right) \boldsymbol{\varphi}_{j}=\mathbf{0} \quad j=1, \ldots, s .
\end{gathered}
$$

The objective function contains the residuals between the target frequency eigenvalues $\left(\bar{\lambda}_{j}\right)$ with both the eigenvalues associated to the system natural frequencies $\left(\lambda_{j}\right)$ and the MRQA $\left(\mu_{j}\right)$. The $a_{j}$ and $b_{j}$ are adequate weighting factors. In the synthesis defined by (6)-(8) we will adjust the design variables and thus the stiffness and mass matrices, such that $\mu_{j}$ and $\lambda_{j}$ will both converge to the natural prescribed target frequency eigenvalues, $\bar{\lambda}_{j}$. For instance, admitting that during the optimization iterations the MRQA will converge to the target frequency eigenvalue $\left(\mu_{j} \rightarrow \bar{\lambda}_{j}\right)$ implies that the mode shape will also converge to $\overline{\boldsymbol{\varphi}}_{j}$; that is, we will have $\boldsymbol{\varphi}_{j} \rightarrow \overline{\boldsymbol{\varphi}}_{j}$.

The problem above is well posed in the sense that it will have a high probability of producing a solution which is the best possible in fulfilling the specified frequencies and mode shapes, but it has no mention of limiting the structural or nonstructural mass mobilized during the solution, and so a probability exists such that a nonoptimal structure in terms of mass and structural weight can be created. Limiting the masses could be an alternative to be exploited, but for now this will be left as an open issue.

The problem statement can be modified with the inclusion of a term containing the structural and nonstructural masses in the objective function so that we can synthesize a system that best fits the prescribed target frequencies and mode shapes but does it with minimum expending of mass. The following new augmented statement of the problem is then proposed with the inclusion of the mass $(M)$ in the 
TABle 1: Target frequencies (Case 1).

\begin{tabular}{lcc}
\hline Mode & $\bar{\lambda}_{j}=\bar{\omega}_{j}^{2}$ & $\bar{f}_{j}(\mathrm{~Hz})$ \\
\hline 1 & 425.2 & 3.28 \\
2 & 8396.6 & 14.58 \\
3 & 47992.1 & 34.87 \\
\hline
\end{tabular}

TABle 2: Target mode shapes (Case 1).

\begin{tabular}{lllllccccccc}
\hline Mode 1 & 0.000 & 0.330 & 0.622 & 0.842 & 0.971 & 1.000 & 0.930 & 0.776 & 0.555 & 0.289 & 0.000 \\
Mode 2 & 0.000 & 0.437 & 0.625 & 0.408 & -0.070 & -0.576 & -0.918 & -1.000 & -0.833 & -0.469 & 0.000 \\
Mode 3 & 0.000 & 0.228 & 0.197 & -0.182 & -0.454 & -0.189 & 0.411 & 0.897 & 1.000 & 0.644 & 0.000 \\
\hline
\end{tabular}

objective function and also range constraints for the frequencies eigenvalues and the MRQAs:

$$
\begin{gathered}
\min M+\sum_{j=1}^{p}\left\{a_{j}\left(\mu_{j}-\bar{\omega}_{j}^{2}\right)^{2}+b_{j}\left(\lambda_{j}-\bar{\omega}_{j}^{2}\right)^{2}\right\}, \\
\text { s.t. } \quad p \bar{\omega}_{j}^{2} \leq \lambda_{j} \leq q \bar{\omega}_{j}^{2} \quad j=1, \ldots, s, \\
p \bar{\omega}_{j}^{2} \leq \mu_{j} \leq q \bar{\omega}_{j}^{2} \quad j=1, \ldots, s, \\
\mu_{j}=\frac{\overline{\boldsymbol{\varphi}}_{j}^{T} \mathbf{K} \overline{\boldsymbol{\varphi}}_{j}}{\overline{\boldsymbol{\varphi}}_{j}^{T} \mathbf{M} \overline{\boldsymbol{\varphi}}_{j}} \quad j=1, \ldots, s, \\
\left(\mathbf{K}-\lambda_{j} \mathbf{M}\right) \boldsymbol{\varphi}_{j}=\mathbf{0} \quad j=1, \ldots, s .
\end{gathered}
$$

The use of the mass augmented objective function of (9) instead of (6) may lead to a compromise solution in which the system mass is minimized but the adjustment between the frequencies and mode shapes to their prescribed values may not be the best. Thus, the range constraints are added ((10)-(11)) so that the problem statement is now given by (9)(13). Range constraints are used instead of strict equality constraints for two reasons. Firstly, satisfaction of equality of frequencies and mode shapes to their prescribed target values may not be possible depending on the design variables used for the synthesis [3]. Also, because the numerical optimization solution tends to be harder for strict equality constraints, even for the case where they are realizable. Here the multipliers $p \leq 1$ and $q \geq 1$ are parameters defining the ranges; for example, $p=1-\delta$ and $q=1+\delta$, where $\delta$ is adjusted during the optimization, departing from say $\delta=0.1$, and closing the range with say $\delta=0.0001$. Experience with simple cases now shows that good solutions can be obtained adjusting the ranges smoothly, by means of solving a sequential optimization with decreasing ranges such that in the $i$ th optimization problem $\delta^{(i)}=r \delta^{(i-1)}$, where $r<1$; for example, $0.1 \leq r \leq 0.5$. Because of the new constraints we can choose the weights $a_{j}$ and $b_{j}$ to be null, so defining a cleaner massonly objective function.

\section{Simply Supported Beam}

A simply supported beam of length $L$, with rectangular cross section $(b, h)$, is shown in Figure 1. It has five concentrated

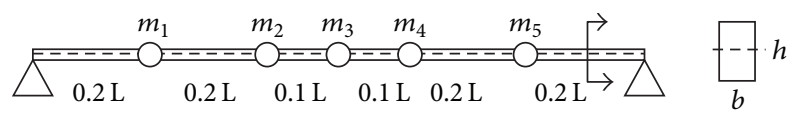

Figure 1: Beam with concentrated masses.

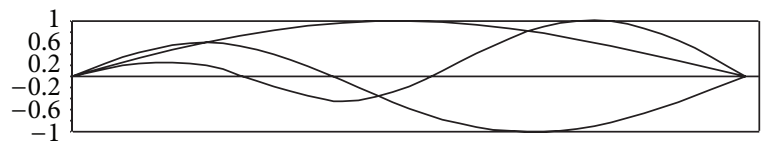

Figure 2: Target mode shapes (Case 1).

masses which are design variables, positioned along its centroidal axis. Also the beam depth, $h$, is a design variable. The beam behavior includes just in-plane bending which obeys the Euler-Bernoulli theory, without rotary inertia effects. The beam natural free vibration analysis is done by the RayleighRitz method, using a sine series for the deflections with ten terms. The length of the beam is $L=5 \mathrm{~m}$, the modulus of elasticity is $E=70 \mathrm{~Pa}$, and the material density is $\rho=$ $2500 \mathrm{~kg} / \mathrm{m}^{3}$.

To optimize the beam for minimum total mass, we have six design variables $\left(h, m_{1}, m_{2}, m_{3}, m_{4}, m_{5}\right)$. Three study cases are defined which differ from each other mainly due to the prescribed target values of frequencies and mode shapes.

3.1. Case 1. In this case the beam section width is $b=50 \mathrm{~mm}$. The target values of frequencies are those in Table 1, while Table 2 has the target mode shapes.

The frequencies and mode shapes of Tables 1 and 2 were chosen to be the same that can be obtained when the beam has the following properties: $h=0.2 \mathrm{~m}, m_{1}=200 \mathrm{~kg}, m_{2}=$ $90 \mathrm{~kg}, m_{3}=50 \mathrm{~kg}, m_{4}=30 \mathrm{~kg}$, and $m_{5}=10 \mathrm{~kg}$; this will be called the reference design.

The target mode shapes are illustrated in Figure 2, where a tendency to symmetry of mode 1 exists while modes 2 and 3 are a mix of symmetric and antisymmetric parts, making the structural synthesis problem harder when compared to a case with only purely symmetric or antisymmetric modes.

The optimization problem is defined by (9)-(13); however we use the simpler objective function corresponding to mass minimization only, that is, with $a_{j}=b_{j}=0, j=1, \ldots, 3$. A sequential optimization solution was implemented with 
TABLE 3: Optimal design variables (Case 1).

\begin{tabular}{lcccccc}
\hline$h(\mathrm{~m})$ & Mass & 1 & 2 & 3 & 5 & \\
\hline 0.075 & $(\mathrm{~kg})$ & 77.61 & 34.07 & 20.11 & 9.89 & \\
\hline
\end{tabular}

TABLE 4: Optimal frequencies and MRQA (Case 1).

\begin{tabular}{lccccc}
\hline Mode & $\bar{\omega}_{j}^{2}$ & $\lambda_{j}$ & \% Error & $\mu_{j}$ & \% Error \\
\hline 1 & 425.2 & 425.2 & 0.01 & 425.2 & 0.01 \\
2 & 8396.6 & 8397.5 & 0.01 & 8395.8 & 0.01 \\
3 & 47992.1 & 47987.3 & 0.01 & 47996.9 & 0.01 \\
\hline
\end{tabular}

TABLE 5: Optimal mode shapes (Case 1).

\begin{tabular}{lllllllccccc}
\hline 1st mode & 0.000 & 0.329 & 0.620 & 0.840 & 0.970 & 1.000 & 0.931 & 0.776 & 0.555 & 0.289 & 0.000 \\
2nd mode & 0.000 & 0.442 & 0.635 & 0.424 & -0.053 & -0.564 & -0.912 & -1.000 & -0.834 & -0.471 & 0.000 \\
3rd mode & 0.000 & 0.243 & 0.210 & -0.182 & -0.467 & -0.205 & 0.406 & 0.902 & 1.000 & 0.647 & 0.000 \\
\hline
\end{tabular}

TABLE 6: Sequential optimization iteration history (Case 1).

(a)

\begin{tabular}{lccccc}
\hline Iter. & $p$ & $q$ & $M(\mathrm{~kg})$ & $\operatorname{Max} g_{\lambda}$ & \multicolumn{1}{c}{ Max $g_{\mu}$} \\
\hline 0 & 0.5 & 2 & 1125. & $5.4 E-01$ & $4.3 E-01$ \\
1 & 0.5 & 2 & 23.59 & $-3.9 E-01$ & $1.3 E-06$ \\
2 & 0.95 & 1.1 & 77.70 & $5.9 E-08$ & $2.3 E-08$ \\
3 & 0.99 & 1.01 & 130.31 & $4.2 E-08$ & $5.4 E-10$ \\
4 & 1.001 & 167.60 & $2.5 E-09$ & $4.9 E-09$ \\
5 & 0.999 & 1.0001 & 188.46 & $8.7 E-10$ & $9.0 E-10$ \\
\hline
\end{tabular}

(b)

\begin{tabular}{lccccccc}
\hline Iter. & $h(\mathrm{~m})$ & $m_{1}(\mathrm{~kg})$ & $m_{2}(\mathrm{~kg})$ & $m_{3}(\mathrm{~kg})$ & $m_{4}(\mathrm{~kg})$ & $m_{5}(\mathrm{~kg})$ & $\sum m_{j}(\mathrm{~kg})$ \\
\hline 0 & 0.2000 & 200 & 200 & 200 & 200 & 0.00 & 0.00 \\
1 & 0.038 & 0.00 & 0.00 & 0.00 & 17.78 & 4.49 & 0.00 \\
2 & 0.058 & 18.31 & 1.01 & 24.54 & 3.46 & 0.00 & 41.59 \\
3 & 0.067 & 46.32 & 14.34 & 21.76 & 7.01 & 0.00 & 88.67 \\
4 & 0.072 & 66.70 & 27.11 & 20.11 & 9.89 & 0.00 & 122.58 \\
5 & 0.075 & 77.61 & 34.07 & & & 141.68 \\
\hline
\end{tabular}

varying $p$ and $q$, having the iteration history presented in Table 6, which will be discussed later.

The optimal values of the six design variables used to minimize the mass and satisfy the target frequencies and mode shapes are presented in Table 3 and correspond to the total mass of $188.46 \mathrm{~kg}$, from which $141.68 \mathrm{~kg}$ is made of concentrated masses. The optimal beam has a depth of $0.075 \mathrm{~m}$. The optimal solution is remarkable from the point of view of reduction of the structural and nonstructural masses, when compared to the reference design where the beam depth is $0.020 \mathrm{~m}$ and the nonstructural mass is $380 \mathrm{~kg}$.

Table 4 has the eigenvalues $\left(\lambda_{j}\right)$ and the values of the MRQA $\left(\mu_{j}\right)$ obtained for the optimal design, where an almost perfect matching is seen with the target values $\bar{\omega}_{j}^{2}$, with deviations around only $0.01 \%$.

Table 5 presents the optimal mode shapes obtained, having a very close agreement with the target modes of Table 2.

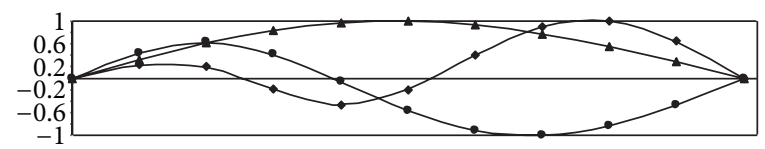

Figure 3: Optimal and target mode shapes (Case 1).

This could be anticipated from the closeness between the MRQA $\left(\mu_{j}\right)$ and $\bar{\omega}_{j}^{2}$ of Table 4 . As a matter of fact, the possibility of achieving this matching is the basis of the solution proposed in this work.

The excellent agreement between optimal and target mode shapes can be seen from Figure 3. The optimal mode shape curves which are marked and the target unmarked can barely be distinguished one from the other. 
TABle 7: Target frequencies (Case 2).

\begin{tabular}{lcc}
\hline Mode & $\bar{\lambda}_{j}=\bar{\omega}_{j}^{2}$ & $\bar{f}_{j}(\mathrm{~Hz})$ \\
\hline 1 & 121.97 & 1.76 \\
2 & 2569.53 & 8.08 \\
3 & 10350.41 & 16.19 \\
\hline
\end{tabular}

TABLE 8: Target mode shapes (Case 2).

\begin{tabular}{lllllccccccc}
\hline Mode 1 & 0.000 & 0.319 & 0.605 & 0.827 & 0.963 & 1.000 & 0.937 & 0.786 & 0.565 & 0.295 & 0.000 \\
Mode 2 & 0.000 & 0.461 & 0.707 & 0.594 & 0.191 & -0.339 & -0.790 & -1.000 & -0.910 & -0.536 & 0.000 \\
Mode 3 & 0.000 & 0.522 & 0.611 & 0.082 & -0.492 & -0.488 & 0.050 & 0.697 & 1.000 & 0.697 & 0.000 \\
\hline
\end{tabular}

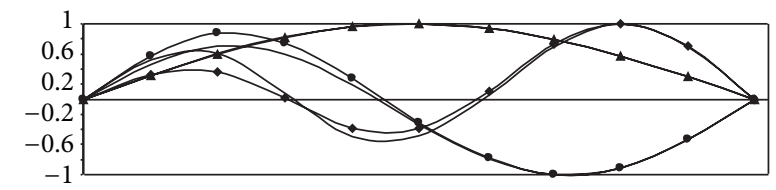

Figure 4: Optimal and target mode shapes (Case 2A).

It is instructive to look at Table 6 with the nice optimization iteration history. Each one of the five iterations corresponds to given values of the constraints range parameters $p$ and $q$, which were quickly found by trial and error. The second row has the initial design and from the third row on the optimal results after the optimization for the corresponding $p$ and $q$ parameters. The total mass presented in the fourth column of Table 6 decays in the first iteration and then grows rapidly with the tightening of $p$ and $q$. The optimization behavior was excellent, allowing the use of rapidly decreasing constraint ranges. Furthermore, the optimization would fail if the tightening of $p$ and $q$ was very sharp or the starting values of $p$ and $q$ were too tight. In the fifth column the maximum constraint violation for the constraints in normalized form of (10) is presented as follows:

$$
\max g_{\lambda}=\max \left(1-\frac{\lambda_{j}}{p \bar{\omega}_{j}^{2}},-1+\frac{\lambda_{j}}{q \bar{\omega}_{j}^{2}}\right) .
$$

Similarly results of the 6th column correspond to (11). The constraints are always satisfied at the optimum of every iteration. The second row of Table 6 has the initial design (iter $=0$ ), with a constraint violation of $54 \%$ associated with $p=0.5$ and $q=2.0$, the values used to solve the first iteration, at the end of which the constraints became satisfied (iter = 1). The solution was obtained with the Solver in Excel.

In the solution presented, the adjustable range constraints were very effective, leading to results as if equality constraints had been imposed, corresponding to $\delta=0.0001$.

3.2. Case 2. Here $b=0.03 \mathrm{~m}$ and the target frequencies are smaller, given in Table 7, with the target mode shapes of Table 8.

The values from Tables 7 and 8 can be obtained from the ref-erence design: $h=0.05 \mathrm{~m}, m_{1}=m_{2}=30 \mathrm{~kg}$, and $m_{3}=$ $m_{4}=m_{5}=10 \mathrm{~kg}$.
TABLE 9: Optimal design variables results (Case 2A).

\begin{tabular}{cccccccc}
\hline$h(\mathrm{~m})$ & Masses & 1 & 2 & 3 & 4 & 5 & $\sum m_{j}$ \\
\hline 0.030 & $(\mathrm{~kg})$ & 5.50 & 1.32 & 5.80 & 0.02 & 0.67 & 13.31 \\
\hline
\end{tabular}

TABLE 10: Optimal frequencies and MRQA (Case 2A).

\begin{tabular}{lccccc}
\hline Mode & $\bar{\omega}_{j}^{2}$ & $\lambda_{j}$ & \% Error & $\mu_{j}$ & \% Error \\
\hline 1 & 121.97 & 124.1 & 1.8 & 124.2 & 1.9 \\
2 & 2569.5 & 2572.4 & 0.1 & 2564.6 & -0.2 \\
3 & 10350.4 & 10345.2 & -0.1 & 10363.2 & 0.1 \\
\hline
\end{tabular}

The problem of (9)-(13) has the same six design variables of Case 1. The beam depth is constrained, with $h \geq 0.03 \mathrm{~m}$. This time both solutions are going to be examined: without (Case 2A) and with (Case 2B) the range constraints.

For the case without range constraints, we use the weights $a_{j}=b_{j}=1 /\left(\bar{\omega}_{j}^{2}\right)$. The optimum design, obtained using the same initial design of Case 1, is given in Table 9.

The optimal frequencies and MRQA obtained in this run are given in Table 10, showing a very satisfactory agreement between the target $\bar{\omega}_{j}^{2}$ and the optimal values of $\lambda_{j}$ and $\mu_{j}$, with errors smaller than $2 \%$.

The optimal mode shapes for the case without range constraints are given in Table 11.

In Figure 4 the optimal mode shapes are depicted in the curves with marks against the unmarked curves of target modes shapes. We can see that the matching is reasonable but not perfect.

The optimal solution for Case $2 \mathrm{~B}$ with range constraints is given in Table 12. It was obtained using as initial design the solution of Case $2 \mathrm{~A}$ and the range constraints with $\delta=$ 0.0001 . As can be observed the solution is very distinct from the one in Table 9 in terms of the design variables values. Besides, the total optimal nonstructural mass is considerably bigger now.

The optimal frequencies and MRQA are given in Table 13, showing an almost perfect matching between the target $\bar{\omega}_{j}^{2}$ and the optimal values of $\lambda_{j}$ and $\mu_{j}$. The comparison between Tables 10 and 13 shows that in Case $2 \mathrm{~B}$ the solution is considerably more precise.

The optimal mode shapes given in Table 14 and depicted in Figure 5 have a close agreement with the target values of 
TABLE 11: Optimal mode shapes (Case 2A).

\begin{tabular}{lccccccccccc}
\hline 1st mode & 0.000 & 0.315 & 0.598 & 0.819 & 0.958 & 1.000 & 0.940 & 0.791 & 0.569 & 0.297 & 0.000 \\
2nd mode & 0.000 & 0.576 & 0.880 & 0.745 & 0.273 & -0.314 & -0.784 & -1.000 & -0.910 & -0.537 & 0.000 \\
3rd mode & 0.000 & 0.329 & 0.368 & 0.016 & -0.382 & -0.392 & 0.108 & 0.729 & 1.000 & 0.700 & 0.000 \\
\hline
\end{tabular}

TABLE 12: Optimal design variables results (Case 2B).

\begin{tabular}{lccccccc}
\hline$h(\mathrm{~m})$ & Masses & 1 & 2 & 3 & 4 & 5 & $\sum m_{j}$ \\
\hline 0.0333 & $(\mathrm{~kg})$ & 7.83 & 5.24 & 5.34 & 1.09 & 1.50 & 21.00 \\
\hline
\end{tabular}

TABLE 13: Optimal frequencies and MRQA (Case 2B).

\begin{tabular}{lccccc}
\hline Mode & $\bar{\omega}_{j}^{2}$ & $\lambda_{j}$ & \% Error & $\mu_{j}$ & \% Error \\
\hline 1 & 121.97 & 121.96 & 0.01 & 121.98 & 0.01 \\
2 & 2569.5 & 2569.3 & 0.01 & 2569.8 & 0.01 \\
3 & 10350.4 & 10349.4 & 0.01 & 10350.4 \\
\hline
\end{tabular}

TABLE 14: Optimal mode shapes (Case 2B).

\begin{tabular}{lccccccccccc}
\hline 1st mode & 0.000 & 0.317 & 0.602 & 0.823 & 0.961 & 1.000 & 0.938 & 0.788 & 0.567 & 0.296 & 0.000 \\
2nd mode & 0.000 & 0.510 & 0.780 & 0.656 & 0.222 & -0.333 & -0.790 & -1.000 & -0.908 & -0.535 & 0.000 \\
3rd mode & 0.000 & 0.419 & 0.479 & 0.046 & -0.432 & -0.433 & 0.088 & 0.719 & 1.000 & 0.699 & 0.000 \\
\hline
\end{tabular}

TABLE 15: Target frequencies (Case 3).

\begin{tabular}{lcc}
\hline Mode & $\bar{\lambda}_{j}=\bar{\omega}_{j}^{2}$ & $\bar{f}_{j}(\mathrm{~Hz})$ \\
\hline 1 & 157.91 & 2 \\
2 & 1934.44 & 7 \\
3 & 8300.34 & 14.5 \\
\hline
\end{tabular}

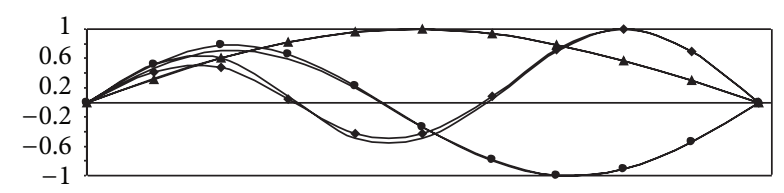

FIgURE 5: Optimal and target mode shapes (Case 2B).

Table 8. It is worth comparing Figure 5, from the case with range constraints, with Figure 4, from the case without them. It is clear that the solution with range constraints results in better adjusted mode shapes.

3.3. Case 3. Now the beam has the same target mode shapes of Case 2 (see Table 8) but different target frequencies according to Table 15.

The target frequencies were set trying to make Case 3 harder than Case 2. This was done by keeping the same target mode shapes of Case 2 but imposing target frequencies which are not scalable from Case 2. With this in mind, Table 15 was created looking at Table 7 and doing the following: increasing the first target frequency from $1.76 \mathrm{~Hz}$ to $2 \mathrm{~Hz}$, decreasing the second target frequency from $8.08 \mathrm{~Hz}$ to $7 \mathrm{~Hz}$, and decreasing the third from $16.19 \mathrm{~Hz}$ to $14.5 \mathrm{~Hz}$.

The optimization now was done by adjusting the parameters $p$ and $q$, resulting in the iterations shown in Table 16. The parameters could be decreased up to a corresponding $\delta=0.03$; values below this would not produce a feasible solution. The values of $p=1-\delta$ and $q=1+\delta$ used to solve the sequential optimization are given, with the remark that for iter $=0$ the values have only the purpose of computing the maximum constraint violations in the initial design.

Table 17 shows that the eigenvalues and the MRQAs for the optimal design could be reasonably adjusted to the target values, with a maximum error of $3 \%$.

The mode shapes also were satisfactorily adjusted as Table 18 shows, when compared to the target values of Table 8.

In Figure 6 continuous lines are the prescribed target mode shapes and the lines with marks are the optimal mode shapes. The first mode is practically the same of the target mode; the second mode seems to be a better fitting to the prescribed target mode than is the third mode. This is the case where to obtain a better adjusting for the second and third modes more design variables would be necessary, such as additional point masses or eventually a beam with variable 
TABLE 16: Sequential optimization iteration history (Case 3).

(a)

\begin{tabular}{lccccc}
\hline Iter & $p$ & $q$ & $M(\mathrm{~kg})$ & $\operatorname{Max} g_{\lambda}$ & \multicolumn{1}{c}{ Max $g_{\mu}$} \\
\hline 0 & 0.9 & 1.1 & 108.75 & $2.08 E-01$ & $2.08 E-01$ \\
1 & 0.9 & 1.1 & 23.03 & $4.95 E-08$ & $-4.44 E-02$ \\
2 & 0.95 & 1.05 & 24.03 & $1.59 E-08$ & $-2.70 E-12$ \\
3 & 0.97 & 1.03 & 186.09 & $1.94 E-08$ & $-7.50 E-10$ \\
\hline
\end{tabular}

(b)

\begin{tabular}{lccccccc}
\hline Iter & $h(\mathrm{~m})$ & $m_{1}(\mathrm{~kg})$ & $m_{2}(\mathrm{~kg})$ & $m_{3}(\mathrm{~kg})$ & $m_{4}(\mathrm{~kg})$ & $m_{5}(\mathrm{~kg})$ & $\sum m_{j}(\mathrm{~kg})$ \\
\hline 0 & 0.0500 & 30.0 & 30.0 & 10.0 & 10.0 & 10.0 & 90.0 \\
1 & 0.0300 & 4.46 & 0.00 & 2.87 & 0.000 & 4.45 & 11.78 \\
2 & 0.0300 & 5.58 & 0.00 & 3.12 & 0.000 & 4.08 & 12.78 \\
3 & 0.0606 & 74.21 & 0.00 & 37.31 & 11.64 & 40.23 & 163.38 \\
\hline
\end{tabular}

TABLE 17: Optimal frequencies and MRQA (Case 3).

\begin{tabular}{lccccc}
\hline Mode & $\bar{\omega}_{j}^{2}$ & $\lambda_{j}$ & \% Error & $\mu_{j}$ & \% Error \\
\hline 1 & 157.9 & 153.2 & 3.0 & 153.9 & 2.5 \\
2 & 1934.4 & 1992.5 & 3.0 & 1969.0 & 1.8 \\
3 & 8300.3 & 8549.4 & 3.0 & 8051.3 & 3.0 \\
\hline
\end{tabular}

TABLE 18: Optimal mode shapes (Case 3).

\begin{tabular}{llllllcccccc}
\hline Mode 1 & 0.000 & 0.318 & 0.601 & 0.818 & 0.955 & 1.000 & 0.947 & 0.803 & 0.583 & 0.306 & 0.000 \\
Mode 2 & 0.000 & 0.587 & 0.909 & 0.792 & 0.346 & -0.224 & -0.722 & -1.000 & -0.961 & -0.580 & 0.000 \\
Mode 3 & 0.000 & 0.397 & 0.430 & -0.080 & -0.737 & -0.970 & -0.455 & 0.435 & 1.000 & 0.763 & 0.000 \\
\hline
\end{tabular}

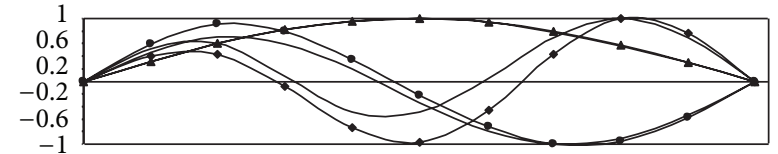

FIgURE 6: Optimal and target mode shapes (Case 3)

depth. However, the important emphasis here is that this result is expected to be very close to the best possible approximate solution for the problem using the same six design variables used for the solution presented.

\section{Concluding Remarks}

A new method that is theoretically consistent was presented for the synthesis of structures with prescribed target frequencies and mode shapes. It avoids the use of eigenvector sensitivity and seems to have excellent convergence potential. Its application to a simple beamled to very encouraging results. It must be tested in more complex synthesis problems for mode and frequency adjusting, where we anticipate that it will provide significant advantages compared to many methods presented in the literature.

\section{Conflict of Interests}

The authors declare that there is no conflict of interests regarding the publication of this paper.

\section{Acknowledgment}

The authors express their gratitude to the Coordenação de Aperfeiçoamento de Pessoal de Nível Superior.

\section{References}

[1] R. A. Canfield, "High-quality approximation of eigenvalues in structural optimization," AIAA Journal, vol. 28, no. 6, pp. 1116$1122,1990$.

[2] V. L. Bond, R. A. Canfield, A. Suleman, and M. Blair, "Aeroelastic scaling of a joined wing for nonlinear geometric stiffness," AIAA Journal, vol. 50, no. 3, pp. 513-522, 2012.

[3] G. M. L. Gladwell, Inverse Problems in Vibration, Martinus Noordhoff, 1986.

[4] E. K. L. Yee and Y. G. Tsuei, "Method for shifting natural frequencies of damped mechanical systems," AIAA journal, vol. 29, no. 11, pp. 1973-1977, 1991.

[5] E. K. L. Yee and Y. G. Tsuei, "Modification of stiffness for shifting natural frequencies of damped mechanical systems," in Proceedings of the ASME Design Technical Conference presented at the 13th Biennial Conference on Mechanical Vibration and Noise, vol. 38, pp. 101-106, September 1991. 
[6] A. J. McMillan and A. J. Keane, "Shifting resonances from a frequency band by applying concentrated masses to a thin rectangular plate," Journal of Sound and Vibration, vol. 192, no. 2, pp. 549-562, 1996.

[7] K.-O. Kim, "A review of mass matrices for eigenproblems," Journal of Computers and Structures, vol. 46, no. 6, pp. 1041-1048, 1993.

[8] D. D. Sivan and Y. M. Ram, "Optimal construction of a massspring system with prescribed modal and spectral data," Journal of Sound and Vibration, vol. 201, no. 3, pp. 323-334, 1997.

[9] G. M. L. Gladwell, "Inverse vibration problems for finiteelement models," Inverse Problems, vol. 13, no. 2, pp. 311-322, 1997.

[10] J. E. Mottershead, "On the zeros of structural frequency response functions and their sensitivities," Mechanical Systems and Signal Processing, vol. 12, no. 5, pp. 591-597, 1998.

[11] S. G. Braun and Y. M. Ram, "Modal modification of vibrating systems: some problems and their solutions," Mechanical Systems and Signal Processing, vol. 15, no. 1, pp. 101-119, 2001.

[12] W. Liangsheng, "Direct method of inverse eigenvalue problems for structure redesign," Journal of Mechanical Design, Transactions of the ASME, vol. 125, no. 4, pp. 845-847, 2003.

[13] Y. M. Ram and J. Caldwell, "Physical parameters reconstruction of a free-free mass-spring system from its spectra," SIAM Journal on Applied Mathematics, vol. 52, no. 1, pp. 140-152, 1992.

[14] A. Kyprianou, J. E. Mottershead, and H. Ouyang, "Structural modification. Part 2: assignment of natural frequencies and antiresonances by an added beam," Journal of Sound and Vibration, vol. 284, no. 1-2, pp. 267-281, 2005.

[15] J. E. Mottershead, M. Ghandchi Tehrani, D. Stancioiu, S. James, and H. Shahverdi, "Structural modification of a helicopter tailcone," Journal of Sound and Vibration, vol. 298, no. 1-2, pp. 366-384, 2006.

[16] G. M. L. Gladwell and M. Movahhedy, "Reconstruction of a mass-spring system from spectral data i: theory," Inverse Problems in Engineering, vol. 1, no. 2, pp. 179-189, 1995.

[17] D. Richiedei, A. Trevisani, and G. Zanardo, "A constrained convex approach to modal design optimization of vibrating systems," Journal of Mechanical Design, Transactions of the ASME, vol. 133, no. 6, Article ID 061011, 2011. 

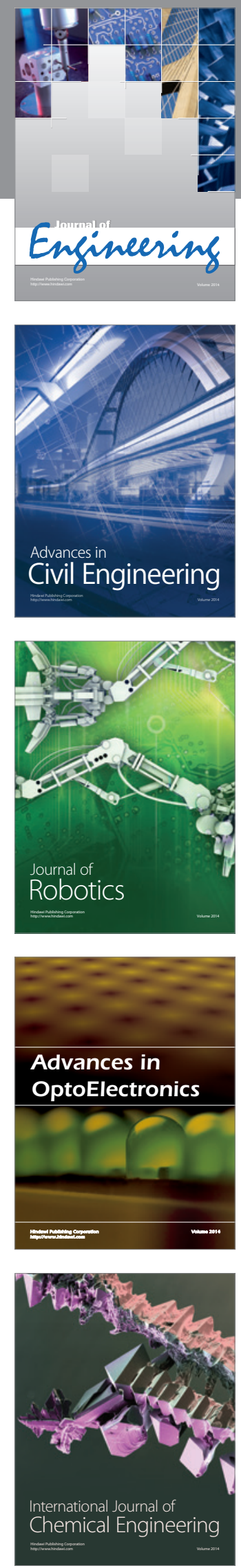

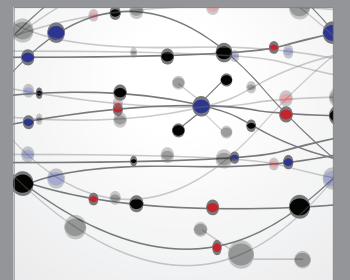

The Scientific World Journal
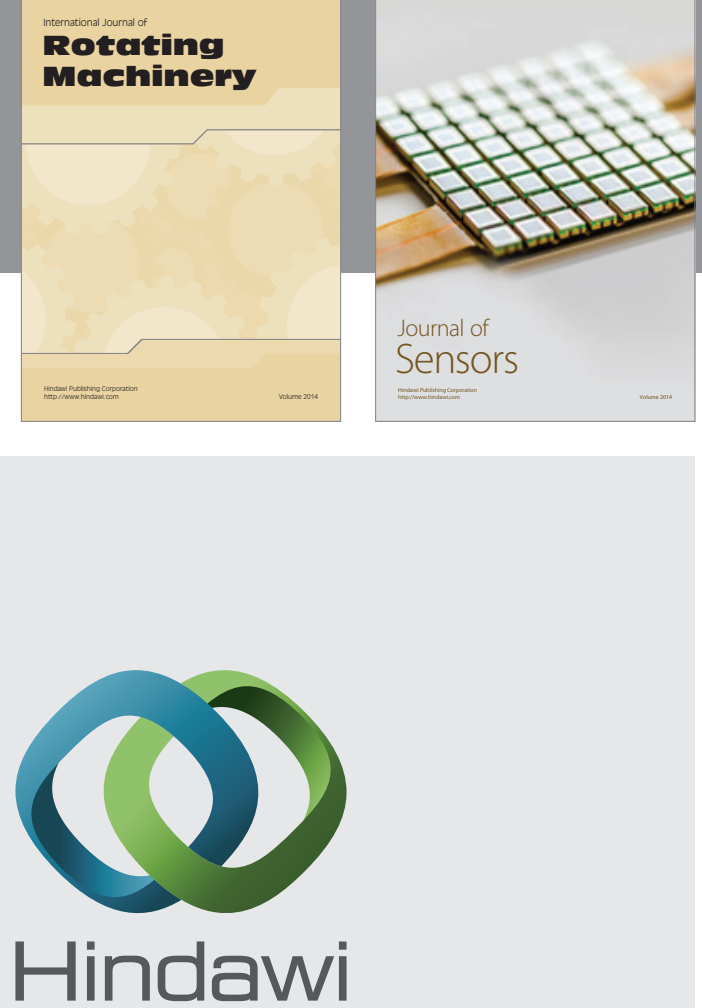

Submit your manuscripts at http://www.hindawi.com
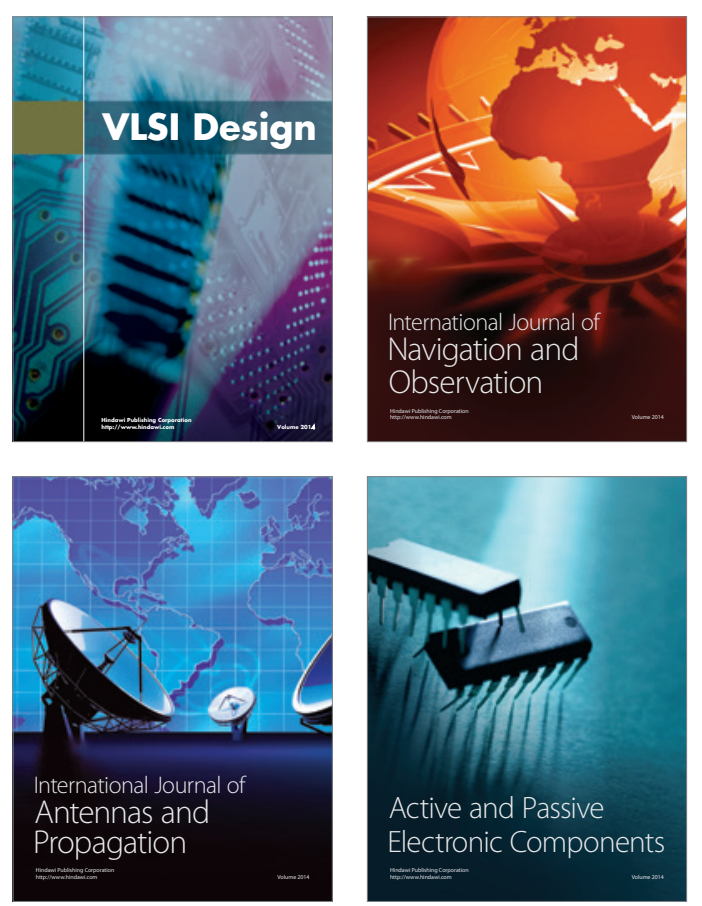
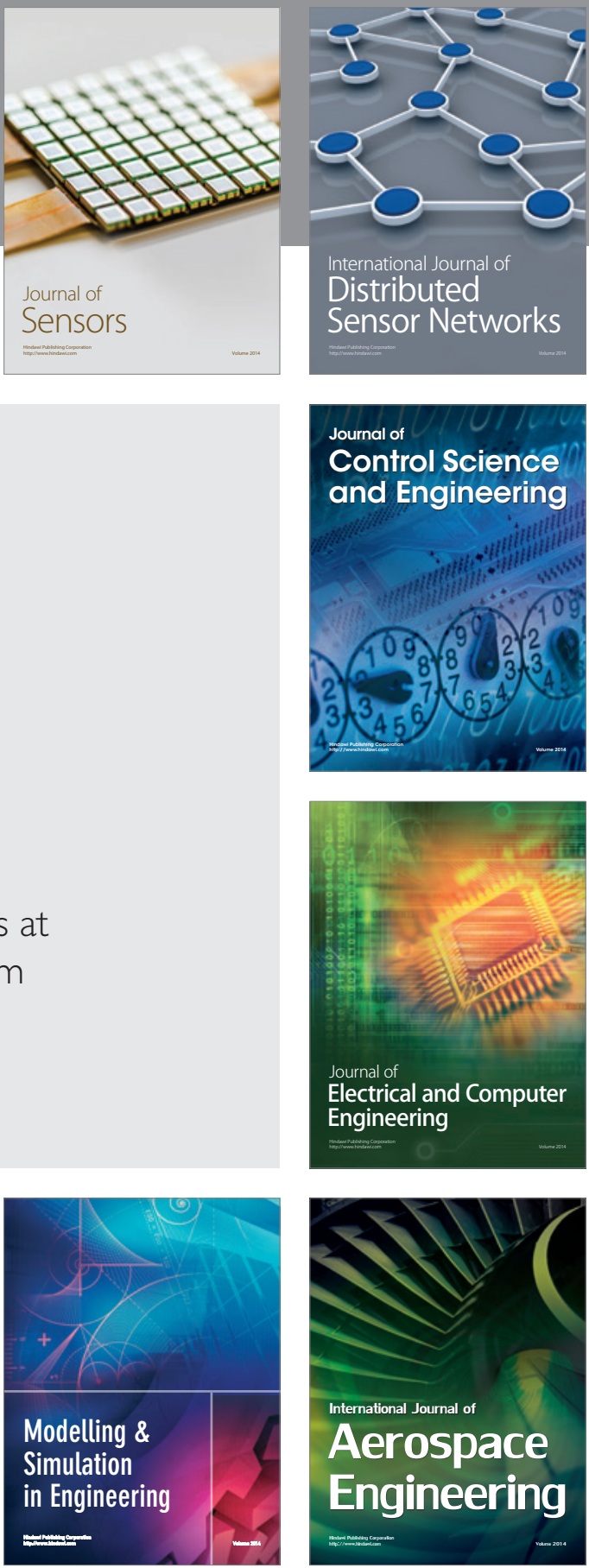

Journal of

Control Science

and Engineering
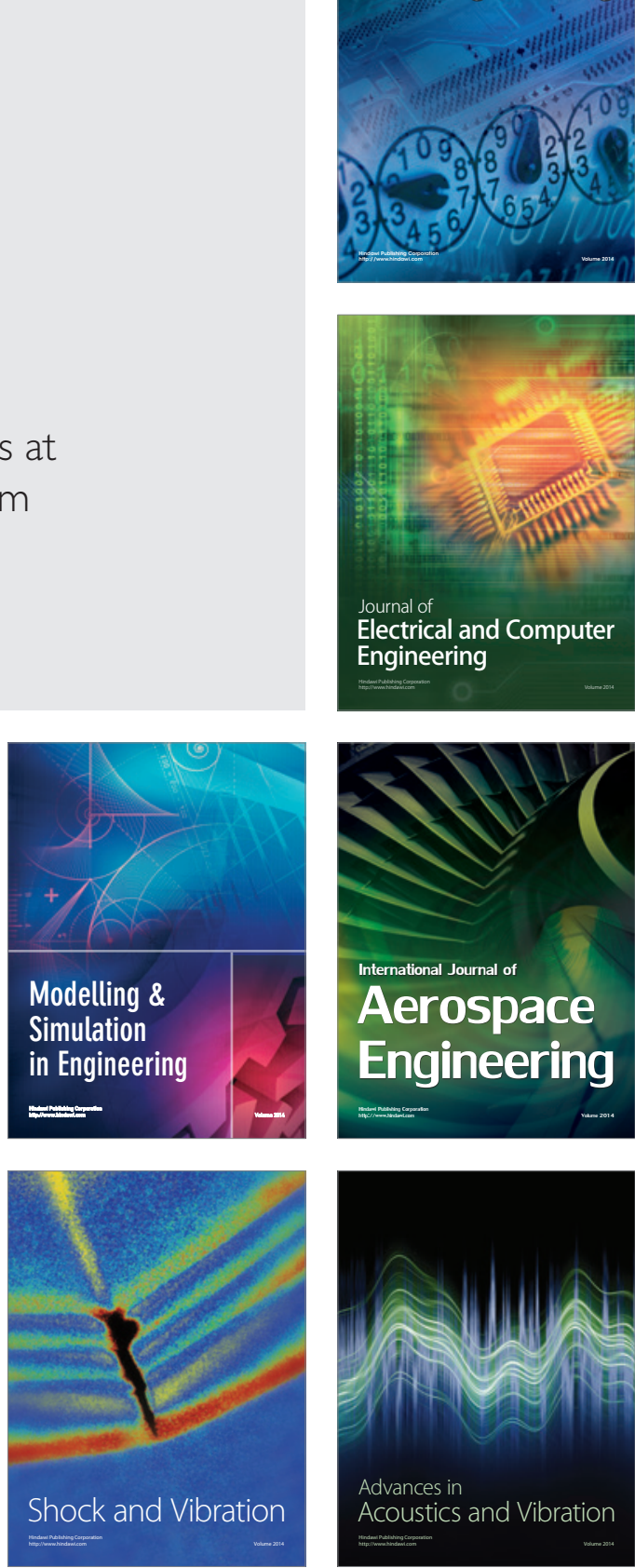